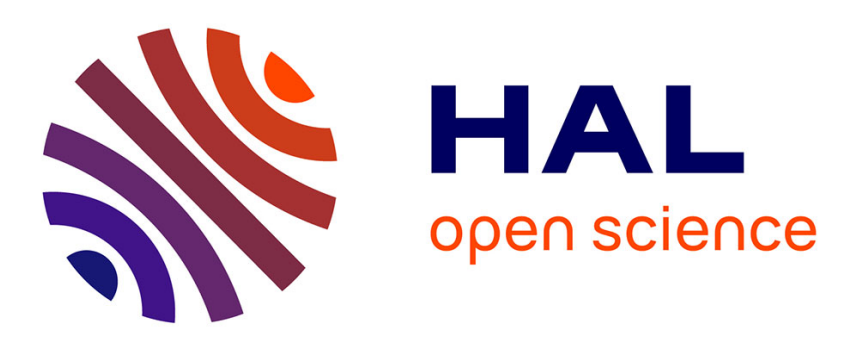

\title{
Noise reduction through joint processing of gravity and gravity gradient data
}

G Pajot, O de Viron, M Diament, M.-F Lequentrec-Lalancette, V Mikhailov

\section{To cite this version:}

G Pajot, O de Viron, M Diament, M.-F Lequentrec-Lalancette, V Mikhailov. Noise reduction through joint processing of gravity and gravity gradient data. Geophysics, 2008, 73 (3), pp.123-134. 10.1190/1.2905222 . insu-01355071

\section{HAL Id: insu-01355071 https://hal-insu.archives-ouvertes.fr/insu-01355071}

Submitted on 22 Aug 2016

HAL is a multi-disciplinary open access archive for the deposit and dissemination of scientific research documents, whether they are published or not. The documents may come from teaching and research institutions in France or abroad, or from public or private research centers.
L'archive ouverte pluridisciplinaire HAL, est destinée au dépôt et à la diffusion de documents scientifiques de niveau recherche, publiés ou non, émanant des établissements d'enseignement et de recherche français ou étrangers, des laboratoires publics ou privés. 


\title{
Noise reduction through joint processing of gravity and gravity gradient data
}

\author{
G. Pajot ${ }^{1}$, O. de Viron ${ }^{2,3}$, M. Diament ${ }^{2}$, M.-F. Lequentrec-Lalancette ${ }^{4}$, and V. Mikhailov ${ }^{2,5}$
}

\begin{abstract}
In mineral and oil exploration, gravity gradient data can help to delineate small-scale features that cannot be retrieved from gravity measurements. Removing high-frequency noise while preserving the high-frequency real signal is one of the most challenging tasks associated with gravity gradiometry data processing. We present a method to reduce gravity and gravity gradient data noise when both are measured in the same area, based on a least-squares simultaneous inversion of observations and physical constraints, inferred from the gravity gradient tensor definition and its mathematical properties. Instead of handling profiles individually, our noise-reduction method uses simultaneously measured values of the tensor components and of gravity in the whole survey area, benefiting from all available information. Synthetic examples show that more than half of the random noise can be removed from all tensor components and nearly all the noise from the gravity anomaly without altering the high-frequency information. We apply our method to a set of marine gravity gradiometry data acquired by Bell Geospace in the Faroe-Shetland Basin to demonstrate its power to resolve small-scale features.
\end{abstract}

\section{INTRODUCTION}

Gravity gradient measurements have been used as a gravity exploration technique since the beginning of the twentieth century. The first instruments, built by Baron Roland von Eötvös (Loránd Eötvös) (Shaw and Lancaster-Jones, 1922), used four independent quantities to measure the horizontal derivatives of the vertical component of the gravity acceleration vector. They were used successfully in field studies, e.g., to map oil fields in Slovakia (Szabó, 1998). Currently, instruments such as the Lockheed Martin gradiometer (Bell et al., 1997) measure five independent gravity derivatives and can retrieve the whole gravity gradient tensor (full-tensor gravity gradiometry, or FTG). In addition to regional surveys, the gravity field and steadystate ocean circulation explorer (GOCE) satellite will be launched by the European Space Agency (ESA) with an onboard gradiometer. Its measurements of the gravity gradient tensor elements covering the whole earth's surface might lead to geoid and gravity-field global models of unprecedented accuracy and spatial resolution (e.g., Klees et al., 2000).

For both GOCE and Lockheed Martin instruments, the derivatives of the gravity vector are measured using pairs of accelerometers, separated from each other by a fixed distance (Hofmeyer and Affleck, 1994). The derivative of the gravity acceleration vector along one direction (defined by one pair of accelerometers) is approximated by the difference between the two measured accelerations, divided by the distance between the accelerometers. When using this system, common-mode nongravitational linear accelerations, sensed identically by two accelerometers, cancel out during data acquisition. Devices and special processing techniques reduce the noise associated with nonlinear accelerations of the craft from sources such as ship rolling or aircraft bumping (Canuto et al., 2003; Dransfield and Lee, 2004).

Measuring gravity gradients retrieves features at a smaller scale than those characterized with similarly retrieved gravity measurements (European Space Agency, 1999; Mumaw, 2004). Thus, removing noise in gradient data without filtering out high-frequency information is a challenge. This requires the development of methods that benefit from simultaneous observations of all FTG components.

To date, gradiometry-data noise-reduction methods developed for airborne and marine surveys have been based on independent analyses of the survey lines and need a priori hypotheses to separate signal from noise. For example, Lyrio et al. (2004) develop a wavelet-filtering method and apply it to tensor components along the survey lines.

\footnotetext{
Manuscript received by the Editor 21 June 2007; revised manuscript received 15 January 2008; published online 2 May 2008.

${ }^{1}$ Formerly Institut de Physique du Globe de Paris, France; presently BRGM, Paris, France. E-mail: g.pajot@brgm.fr.

${ }^{2}$ Institut de Physique du Globe de Paris, France. E-mail: deviron@ipgp.jussieu.fr; diament@ipgp.jussieu.fr; valentin@ipgp.jussieu.fr

${ }^{3}$ Université Paris-Diderot (UFR STEP), Paris, France.

${ }_{5}^{4}$ Service Hydrographique et Océanographique de la Marine, Brest, France.E-mail: mflqc@ @ shom.fr.

${ }^{5}$ Institute of Physics of the Earth, Moscow, Russia.

(C) 2008 Society of Exploration Geophysicists. All rights reserved.
} 
This method assumes that a large number of finer-scale coefficients of the wavelet reconstruction are pure noise and that the larger-scale coefficients are pure signal. Lyrio et al. (2004) propose a method of determining which scales must be filtered out and, for the remaining scales, the minimum value of the wavelet coefficients retained. The remaining nonzero coefficients are then used to estimate the filtered signal.

The assumption that noise and signal have different energy in real cases, although reasonable, cannot be demonstrated strictly. Moreover, wavelet thresholding remains a filtering method. As Lyrio et al. (2004) point out, short-scale features associated with targets of interest are preserved, but part of the removed field still can be associated with shallow sources. While et al. (2006) characterize the spectral content of gravity gradients using 2D information; they can determine a cutoff wavelength above which the signal can be considered nongeophysical. However, this method fails when applied to real data because, according to the authors, the assumptions made on certain properties of the source might not be true for real data.

Recent methods (Mikhailov et al., 2007) are based on the fact that simultaneously measured gravity gradients are related physically to each other to locate the sources. What we propose is in the same spirit - a noise-reduction method based only on physical considerations. Being the successive derivatives of the same potential, gravity and gravity gradients are related to each other by differential equations. We keep as signal the part of the data consistent with these differential equations. The inconsistent part then is considered to be pure noise.

For this study, we used a data set with nearly parallel survey lines and perpendicular control lines, in the horizontal plane, and with simultaneous gravity and gravity gradiometry measurements. Consequently, all the algorithms and tests were developed under those assumptions. In particular, we established the differential relationships for a survey during which gravity and gravity gradients are measured simultaneously and leveled to a common plane (e.g., marine gravity gradiometry surveys). Although the method can be generalized easily, we did not test the performance of the generalized method. We inverted all of the observation equations together with the differential equations. This yielded an enhanced data set using all available information.

The first section of our article addresses the mathematical formalism of the method. In the second section, we demonstrate on synthetic data the capability of our method to remove random noise, correlated or not, while preserving short-scale patterns. In that prospect, our method and filtering can be used complementarily as long as we do not have additional physical constraints. In the third section, we apply the method to real marine gravity gradiometry data collected by Bell Geospace in the Faroe-Shetland Basin. Finally, we discuss the relevance of our method to mineral exploration and satellite data processing.

\section{METHOD}

\section{Equations}

Gravity gradiometry data are measurements of the derivatives of the components of the gravity vector $\mathbf{g}=\left(g_{x}, g_{y}, g_{z}\right)$ in three orthogonal directions of space $(x, y, z)$. These derivatives are the components of the gravity gradient tensor, which we denote by

$$
\begin{gathered}
\mathbf{T}=\left[\begin{array}{ccc}
T_{x x} & T_{x y} & T_{x z} \\
T_{y x} & T_{y y} & T_{y z} \\
T_{z x} & T_{z y} & T_{z z}
\end{array}\right], \quad \text { where } \\
T_{\alpha \beta}=\frac{\partial g_{\alpha}}{\partial \beta}=\partial_{\beta} g_{\alpha} \quad \forall\{\alpha, \beta\} \subset\{x, y, z\} .
\end{gathered}
$$

The gravity vector components are first-order derivatives of a potential function $V$, which obeys Laplace's equation in free space. Gravity gradients are thus second-order derivatives of the same potential function $V$. Therefore, in free space, $\mathbf{T}$ is symmetric:

$$
T_{\alpha \beta}=\frac{\partial}{\partial \alpha} \frac{\partial V}{\partial \beta}=\frac{\partial}{\partial \beta} \frac{\partial V}{\partial \alpha}=T_{\beta \alpha}, \quad \forall\{\alpha, \beta\} \subset\{x, y, z\} .
$$

The trace of $\mathbf{T}$ is zero:

$$
\sum_{\alpha \in\{x, y, z\}} T_{\alpha \alpha}=\nabla^{2} V=0 .
$$

Because of equations 2 and 3, only five of the nine components of the tensor are independent quantities. Simultaneous measurements of five quantities at every point are enough to recover the whole tensor (FTG). In marine FTG surveys, the gravity gradients and $g_{z}$ are measured simultaneously, with $g_{z}$ measured independently from the gradients. Our theoretical work is based on simultaneous measurements of $T_{x x}, T_{x y}, T_{x z}, T_{y y}, T_{y z}$, and $g_{z}$. It can be extended to any case in which those quantities can be computed from other measurements, with independent errors.

By definition of $\mathbf{T}$, the following linear differential equation system holds:

$$
\left\{\begin{array}{l}
\partial_{y} T_{x x}=\partial_{x} T_{x y} \\
\partial_{y} T_{x y}=\partial_{x} T_{y y} \\
\partial_{y} T_{x z}=\partial_{x} T_{y z} \\
\partial_{y} g_{z}=T_{y z} \\
\partial_{x} g_{z}=T_{x z} \\
\partial_{z} T_{x x}=\partial_{x} T_{x z} \\
\partial_{z} T_{x y}=\partial_{x} T_{y z} \\
\partial_{z} T_{y y}=\partial_{y} T_{y z} \\
\partial_{z} g_{z}=T_{z z}
\end{array}\right.
$$

We consider that measurements are made at a constant $z$-level. We do not consider upward-continued data; therefore, we drop the last four equations of system 4 . We are left with two independent subsystems:

$$
\left\{\begin{array}{l}
\partial_{y} T_{x x}=\partial_{x} T_{x y} \\
\partial_{y} T_{x y}=\partial_{x} T_{y y}
\end{array} \quad\right. \text { (horizontal system) }
$$

and

$$
\left\{\begin{array}{l}
\partial_{y} T_{x z}=\partial_{x} T_{y z} \\
\partial_{x} g_{z}=T_{x z} \\
\partial_{y} g_{z}=T_{y z}
\end{array} \quad\right. \text { (vertical system) }
$$


In the second subsystem, only two equations of the three are independent. We keep the first two, reducing system 6 to

$$
\left\{\begin{array}{l}
\partial_{y} T_{x z}=\partial_{x} T_{y z} \\
\partial_{x} g_{z}=T_{x z}
\end{array} \quad\right. \text { (vertical system). }
$$

Ideal noise-free measurements of $T_{\alpha \beta}$ and $g_{z}$ would satisfy the conditions imposed by systems 5 and 7 at any measurement point. Random noise, on the contrary, would not follow these equations. We use systems 5 and 7 as physical constraints that must be satisfied by noise-free measurements, in combination with observation equations, to reduce the random noise in the data. In other words, we compute $T_{x x}, T_{x y}, T_{x z}, T_{y y}, T_{y z}$, and $g_{z}$ that at each measurement point satisfy optimally both systems 5 and 7 and the observation equations

$$
\left\{\begin{array}{l}
T_{\alpha \beta}=T_{\alpha \beta}^{m}, \quad \alpha \beta \in\{x x, x y, y y, x z, y z\} \\
g_{z}=g_{z}^{m},
\end{array},\right.
$$

where the superscript $m$ denotes the measured value. Note that Laplace's equation (zero trace, equation 3) usually is used to relate the tensor components to each other to improve signal quality (Hammond, 1999) while acquiring FTG data.

Our method differs from previous work because it uses differential equations to relate the components to each other. In particular, our method requires that we provide an adequate way to compute the derivatives of the components. This problem is addressed later in this paper.

\section{Least-squares formulation of the problem}

We assume henceforward that the measurement errors follow a Gaussian or close to Gaussian distribution. We simultaneously solve observation equations 8 with physical-constraint equations 5 and 7 to reduce noise in the data. Practically, we compute the values of $T_{x x}$, $T_{x y}, T_{x z}, T_{y y}, T_{y z}$, and $g_{z}$ so that they minimize the sum over all data points of the squared misfits $d_{\alpha}^{\text {obs }}$ between computed values and observations, defined at each data point $i$ by

$$
d_{\alpha}^{\mathrm{obs}}(i)=\left|T_{\alpha}(i)-T_{\alpha}^{m}(i)\right|,
$$

where $T_{\alpha}^{m}(i)$ is the value of a component (tensor component or $g_{z}$ ) measured at one point $i$, and $T_{\alpha}(i)$ is the value of the same component retrieved by our method at the same point.

The computed values simultaneously must satisfy the physical constraint equations, which means that in addition to minimizing the sum of the squared misfits $d_{\alpha}^{\text {obs }}$ to the measured values, they also must minimize the sum of the squared quantities $d_{\gamma \delta}^{\text {eq }}$, defined by

$$
d_{\gamma \delta}^{\mathrm{eq}}(i)=\left|\partial_{x} T_{\gamma}(i)-\partial_{y} T_{\delta}(i)\right|,
$$

where $T_{\gamma}(i)$ and $T_{\delta}(i)$ stand for tensor components involved in one equation of the horizontal system 5 or in the first equation of the vertical system 7. We also minimize, from the last equation of the vertical system 7, the sum of the squared quantities $d^{\text {eq }}$, defined by

$$
d^{\mathrm{eq}}(i)=\left|\partial_{x} g_{z}(i)-T_{x z}(i)\right| .
$$

For Gaussian errors, finding the minimum variance solution to this problem is equivalent to minimizing the sum of the square residuals $\left(d^{\text {obs }}\right)^{2}+\left(d^{\text {eq }}\right)^{2}$. Consequently, for the horizontal system 5 , we compute values of $T_{x x}, T_{x y}$, and $T_{y y}$ that minimize the sum

$$
\begin{aligned}
R^{h}= & \sum_{\text {datapoints }}\left(\left|T_{x x}-T_{x x}^{m}\right|^{2}+\left|T_{x y}-T_{x y}^{m}\right|^{2}+\left|T_{y y}-T_{y y}^{m}\right|^{2}\right. \\
& \left.+\left|\partial_{y} T_{x x}-\partial_{x} T_{x y}\right|^{2}+\left|\partial_{y} T_{x y}-\partial_{x} T_{y y}\right|^{2}\right)
\end{aligned}
$$

For system 7 , we compute values of $T_{x z}, T_{y z}$, and $g_{z}$ that minimize the sum

$$
\begin{aligned}
R^{v}= & \sum_{\text {datapoints }}\left(\left|T_{x z}-T_{x z}^{m}\right|^{2}+\left|T_{y z}-T_{y z}^{m}\right|^{2}+\left|g_{z}-g_{z}^{m}\right|^{2}\right. \\
& \left.+\left|\partial_{y} T_{x z}-\partial_{x} T_{y z}\right|^{2}+\left|\partial_{x} g_{z}-T_{x z}\right|^{2}\right)
\end{aligned}
$$

Quantities in equation 13 are set to be dimensionless, as explained below. By nature, the method is not designed to remove any bias.

\section{Computing the derivatives}

To compute the enhanced values of the components, we need a way to compute their derivatives. Most of the numerical differentiation schemes express derivatives as linear combinations of data distributed on a lattice. This discretization process is associated with an error becoming larger when the distance between two data points increases. Assuming that the data are distributed on a grid with regular steps $\Delta_{x}$ and $\Delta_{y}$ along $x$ - and $y$-directions, we basically adopt two derivative schemes.

The first is to compute second-order centered finite differences. The derivative $\partial_{x} T_{\alpha}\left(x_{i}, y_{j}\right)$ of one component $T_{\alpha}$ is approximated at point $\left(x_{i}, y_{j}\right)$ by

$$
\partial_{x} T_{\alpha}(i, j) \approx \frac{T_{\alpha}\left(x_{i+1}, y_{j}\right)-T_{\alpha}\left(x_{i-1}, y_{j}\right)}{2 \Delta_{x}},
$$

where $\left(x_{i+1}, y_{j}\right)$ and $\left(x_{i-1}, y_{j}\right)$ are neighboring data points of $\left(x_{i}, y_{j}\right)$ in the $x$-direction, both separated from $\left(x_{i}, y_{j}\right)$ on the computing grid by $\Delta_{x}$. Similar expressions hold for the derivatives along the $y$-direction.

The second scheme is to compute from several neighboring points a low-degree polynomial fit of the data in the direction of the derivative and to use the analytical derivative of this polynomial function to approximate the derivative of the component. (Details are given in Appendix A.) Such an approximation is necessary when data are sampled much more densely in one direction than in the other. Using this approximation helps to prevent noise enhancement resulting from the derivative computation (here, this effect is considered much larger than the discretization error).

Then derivative $\partial_{x} T_{\alpha}\left(x_{i}, y_{j}\right)$ of one component $T_{\alpha}$ in the $x$-direction is approximated at point $\left(x_{i}, y_{j}\right)$ by

$$
\partial_{x} T_{\alpha}\left(x_{i}, y_{j}\right) \approx \sum_{m=i-N}^{i+N} \beta_{m} T_{\alpha}\left(x_{m}, y_{j}\right),
$$

where coefficients $\beta_{m}$ are computed according to the method described in Appendix A. Typically, the number $2 N+1$ of points from which the polynomial approximation is computed is chosen such that the distance $\Delta=x_{i+N}-x_{i-N}$ equals $\Delta_{y}$. We use a similar expression when computing the derivative along the $y$-direction.

The finite-difference method, albeit simplistic, yields results as good as the more sophisticated polynomial method as long as the data are distributed evenly. It is much faster; therefore, we use it in all the tests with evenly distributed data. We do not need data to be distributed along profiles parallel to the $x$ - and $y$-axes. Derivatives can 
be computed in the two ways mentioned above using the true coordinates of the data points. However, for simplicity, we present our results for data distributed on regular grids. We address the problem of irregular data sampling later, when applying our method to real marine gravity gradiometry data.

\section{Matrix formulation}

The derivatives of one tensor component are expressed as linear combinations of the values of the same component at neighboring points. This allows us to write the problem of minimizing residuals in equations 12 and 13 in matrix form. We denote $\mathbf{A}$ as the matrix of normal equations (physical constraint and observation equations), $\mathbf{x}$ as the vector containing the unknowns (i.e., the enhanced values of the tensor components and $g_{z}$ ), and $\mathbf{b}$ as the observation vector. The system of equations

$$
\mathbf{A x}=\mathbf{b}
$$

is overdetermined. We compute the values of $T_{x x}, T_{x y}, T_{x z}, T_{y y}, T_{y z}$, and $g_{z}$ that minimize the $L^{2}$-norm of the vector $\mathbf{A x}-\mathbf{b}$.

Two kinds of equations are described by A: observation and physical constraint. Matrix A is made of two separate blocks. One block has observation equations of the type given in equation 8 . The corresponding block of the normal matrix is diagonal, and the associated block of vector $\mathbf{b}$ is the set of observations. The second block has physical constraint equations 5 and 7. Equations of the form

$$
\frac{\partial}{\partial x} T_{y \alpha}\left(x_{i_{0}}, y_{i_{0}}\right)=\frac{\partial}{\partial y} T_{x \alpha}\left(x_{i_{0}}, y_{i_{0}}\right)
$$

can be written as

$$
\sum_{i \in \mathcal{V}_{x}\left(i_{0}\right)} a_{i} T_{y \alpha}\left(x_{i}, y_{i_{0}}\right)-\sum_{i \in \mathcal{V}_{y}\left(i_{0}\right)} b_{i} T_{x \alpha}\left(x_{i_{0}}, y_{i}\right)=0
$$

where $\mathcal{V}_{x}\left(i_{0}\right)$ and $\mathcal{V}_{y}\left(i_{0}\right)$ are sets of indices denoting coordinates of neighboring points of $\left(x_{i_{0}}, y_{i_{0}}\right)$. The number and positions of the neighboring points depend on the method chosen to compute the derivatives, as do the values of coefficients $a_{i}$ and $b_{i}$. Similar expressions are derived for equations 7 involving $g_{z}$.

For instance, using a centered second-order finite-difference scheme, the first equation of system 5 becomes

$$
\begin{gathered}
\frac{1}{\Delta_{x}} T_{x y}\left(x_{i+1}, y_{i}\right)-\frac{1}{\Delta_{x}} T_{x y}\left(x_{i-1}, y_{i}\right)-\frac{1}{\Delta_{y}} T_{x x}\left(x_{i}, y_{i+1}\right) \\
+\frac{1}{\Delta_{y}} T_{x x}\left(x_{i}, y_{i-1}\right)=0 .
\end{gathered}
$$

The associated block of the normal matrix is not far from diagonal, and the corresponding components of $\mathbf{b}$ are zeros.

Matrix A is sparse. Many algorithms solve least-squares inversion quickly and with fair stability (e.g., Paige and Saunders, 1982). When expressed in SI units [usual units are the Eötvös unit for gravity gradients $\left(1 \mathrm{E}=10^{-9} \mathrm{~s}^{-2}\right)$ and milligals for gravity (1 $\left.\left.\mathrm{mGal}=10^{-5} \mathrm{~m} / \mathrm{s}^{2}\right)\right], g_{z}$ and tensor component values associated with the same geology might differ by several orders of magnitude. This affects the efficiency of the inversion algorithm and overweights the impact of some of the equations.

To overcome this problem, equation systems 5 and 7 are transformed so the equations involve only quantities with no physical di- mension and are of first-order magnitude. Instead of solving for $g_{z}$ and tensor components $T_{\alpha \beta}$, we solve for quantities

$$
\left\{\begin{array}{l}
g_{z}^{\prime}=\frac{1}{g_{0}} g_{z} \\
T_{\alpha \beta}^{\prime}=\frac{\Delta_{0}}{g_{0}} T_{\alpha \beta}
\end{array}\right.
$$

where $g_{0}$ is the standard deviation of the value of $g_{z}$ in the survey area and $\Delta_{0}$ is the diameter of the survey area. Intervals $\Delta_{x}$ and $\Delta_{y}$ between points along the $x$ - and $y$-directions become $\Delta_{x}^{\prime}$ and $\Delta_{y}^{\prime}$, respectively, where

$$
\left\{\begin{array}{l}
\Delta_{x}^{\prime}=\frac{\Delta_{x}}{\Delta_{0}} \\
\Delta_{y}^{\prime}=\frac{\Delta_{y}}{\Delta_{0}}
\end{array} .\right.
$$

This procedure improves the efficiency of the algorithm for system 7. For example, replacing $T_{x x}$ and $T_{x y}$ by $\Delta_{0} / g_{0} T_{x x}^{\prime}$ and $\Delta_{0} / g_{0} T_{x y}^{\prime}$, respectively, and $\Delta_{x}$ and $\Delta_{y}$ by $\Delta_{0} \Delta_{x}^{\prime}$ and $\Delta_{0} \Delta_{y}^{\prime}$ transforms equation 19 into

$$
\begin{aligned}
& \frac{1}{\Delta_{x}^{\prime}} T_{x y}^{\prime}\left(x_{i+1}, y_{i}\right)-\frac{1}{\Delta_{x}^{\prime}} T_{x y}^{\prime}\left(x_{i-1}, y_{i}\right)-\frac{1}{\Delta_{y}^{\prime}} T_{x x}^{\prime}\left(x_{i}, y_{i+1}\right) \\
& +\frac{1}{\Delta_{y}^{\prime}} T_{x x}^{\prime}\left(x_{i}, y_{i-1}\right)=0
\end{aligned}
$$

The finite-difference form of the last equation of system 7 becomes

$$
\frac{1}{\Delta_{x}^{\prime}} g_{z}^{\prime}\left(x_{i+1}, y_{i}\right)-\frac{1}{\Delta_{x}^{\prime}} g_{z}^{\prime}\left(x_{i-1}, y_{i}\right)-T_{x z}^{\prime}\left(x_{i}, y_{i}\right)=0 \text {. }
$$

In our study, we normalize data to account for the amplitude difference of the different measured quantities. This can be extended to account for a priori knowledge about the quality of each measurement (e.g., if the variance/covariance matrix is known).

\section{TESTS ON SYNTHETIC DATA}

In this section, tests are performed using synthetic data computed on regular square grids.

\section{Estimator for noise reduction}

Denoting $X$ as the synthetic value of a tensor component or of $g_{z}$ prior to adding noise, $X_{n}$ the value when noise is added, and $X_{r}$ the result of our least-squares fit, we determine the performance of the noise-reduction method by estimating for each component the noise-reduction factor:

$$
\lambda=\frac{\operatorname{var}\left(X_{n}-X\right)-\operatorname{var}\left(X_{r}-X\right)}{\operatorname{var}\left(X_{n}-X\right)}
$$

where var is a discrete realization of the variance computed by using all of the values on the grid. A positive value of $\lambda$ indicates that the noise has been reduced, and the magnitude of $\lambda$ is the ratio of the eliminated noise. Values close to one indicate a high percentage of 
the noise has been removed; near-zero values indicate poor performance of the method.

\section{Sensitivity to the nature and magnitude of noise}

We first describe the sensitivity of our method to data resolution and noise level using Gaussian white noise and autocorrelated noise. To this end, using formulas derived by Okabe (1979), we compute synthetic gravity gradients and $g_{z}$ corresponding to a structure made of three rectangular prisms; the characteristics are listed in Table 1 and illustrated in Figure 1. This structure combines bodies at different depths and with different spatial extents, which allows us to test the efficiency of our noise-reduction method for a broad range of spatial scales. The three bodies could represent a large, deep basement (prism 1), a shallow anomaly (prism 2), and a thin dyke (prism 3).

The synthetic signal is seen on the left panels of Figures 2 and 3.

\section{Gaussian white noise}

Tests are performed with white Gaussian noise of standard deviation $\sigma$ (in Eötvös for the tensor components, in milligals for $g_{z}$ ), which is set as a percentage of the maximum peak-to-peak amplitude of the original (noise-free) component.

\section{Data resolution}

We add noise to the synthetic data with $\sigma$ equal to $10 \%$ of the maximum peak-to-peak amplitude of the components and compare the noise-reduction results at different grid resolutions (grid steps). These results are shown in Table 2 and are illustrated in Figures 2 and 3 . Increasing grid resolution improves the results. In particular, $\lambda$ reaches a value that differs from one by less than five per mil, indicating that the noise-reduction technique removes more than $99 \%$ of the noise on $g_{z}$. In addition, more than half of the noise is removed from the tensor components.

However, as the grid step decreases, $\lambda$ tends asymptotically to a maximum value $\lambda_{T}^{\max }$ that depends on the component $T$ (tensor component, or $g_{z}$ ) under consideration. These $\lambda^{\max }$ values are such that, defining for each system the average value $\lambda_{\lim }$ by

$$
\left\{\begin{array}{l}
\lambda_{\lim }^{h}=\frac{\lambda_{T_{x x}}^{\max }+\lambda_{T_{x y}}^{\max }+\lambda_{T_{y y}}^{\max }}{3} \text { for system } 5 \\
\lambda_{\lim }^{v}=\frac{\lambda_{T_{x z}}^{\max }+\lambda_{T_{y z}}^{\max }+\lambda_{g_{z}}^{\max }}{3} \text { for system } 7
\end{array},\right.
$$

we have

$$
\left\{\begin{array}{l}
\lambda_{\lim }^{h} \approx \frac{2}{3} \\
\lambda_{\lim }^{v} \approx \frac{2}{3}
\end{array}\right.
$$

The two-thirds ratio probably is linked with the ratio between the number of independent equations (two) and the number of unknowns (three).
To confirm this conjecture, we performed tests removing one equation. Removing one equation leaves one equation with two unknowns, leading to $\lambda_{\lim }^{h}=\lambda_{\lim }^{v} \approx 1 / 2$. The two systems do not have the same behavior, however. For system 5 , both tensor components

Table 1. Characteristics of the three rectangular prisms considered in the synthetic model. Positions of the centers of the top sides are given in the -plane of a Cartesian frame $(O, x, y, z)$. Prism 3 is rotated in the $(x, y)$-plane around its center.

\begin{tabular}{lccccc}
\hline Prism & $\begin{array}{c}\text { Dimensions } \\
x \times y \times z \\
(\mathrm{~km}) \times(\mathrm{km}) \times(\mathrm{km})\end{array}$ & $\begin{array}{c}\text { Top side } \\
\text { center }(x, y) \\
(\mathrm{km}, \mathrm{km})\end{array}$ & $\begin{array}{c}\text { Top side } \\
\text { depth } \\
(\mathrm{km})\end{array}$ & $\begin{array}{c}\text { Rotation } \\
\text { angle } \\
(\text { radians })\end{array}$ & $\begin{array}{c}\text { Density } \\
\text { contrast } \\
\left(\mathrm{kg} / \mathrm{m}^{3}\right)\end{array}$ \\
\hline 1 & $30 \times 5 \times 8$ & $(25.0,17.5)$ & 3.0 & 0 & +500 \\
2 & $3 \times 3 \times 1$ & $(15.0,25.0)$ & 0.5 & 0 & -300 \\
3 & $1 \times 20 \times 7.5$ & $(40.8,25.1)$ & 0.5 & $-\pi / 4$ & +300 \\
\hline
\end{tabular}

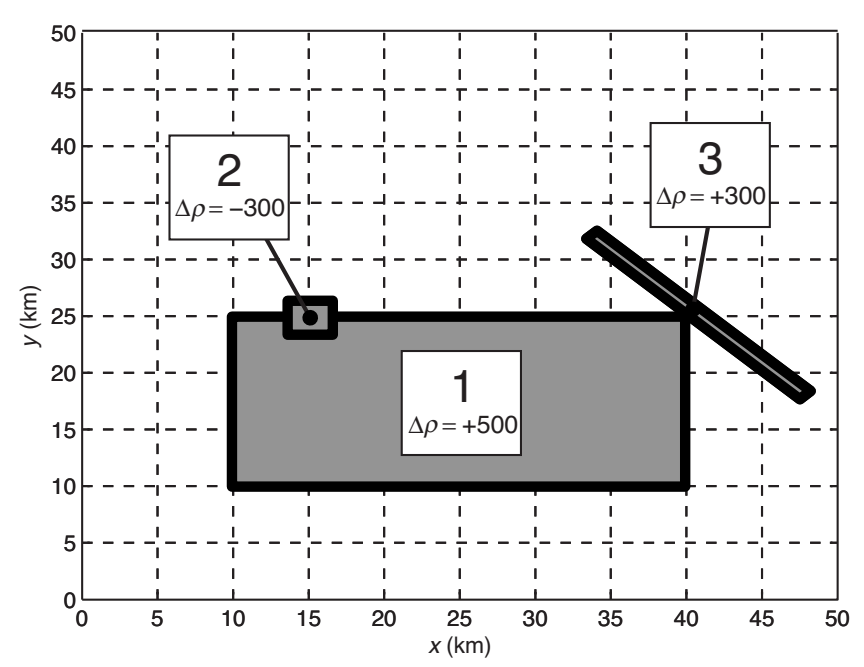

Figure 1. Projection, onto the $(x, y)$-plane, of the three blocks described in Table 1 . Density contrasts $\Delta \rho$ are in kilograms per cubic $\operatorname{meter}\left(\mathrm{kg} / \mathrm{m}^{3}\right)$.
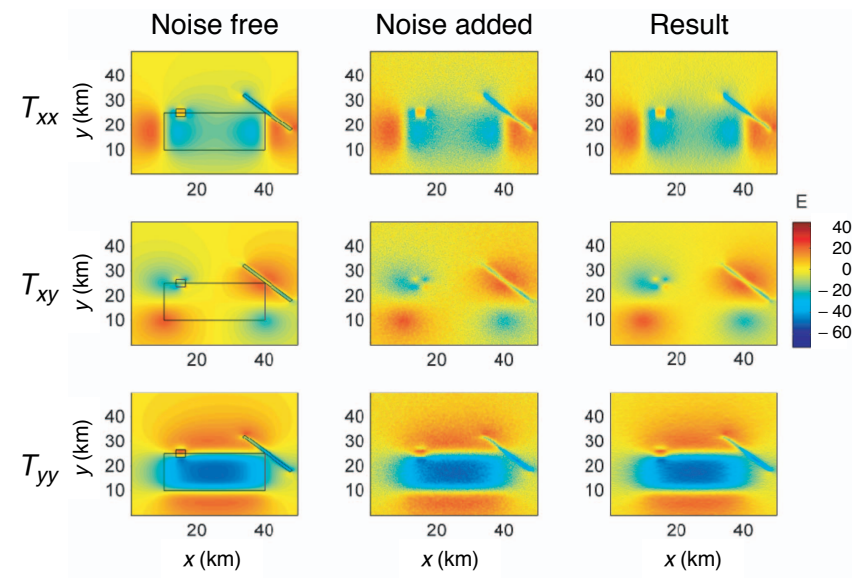

Figure 2. Noise-reduction results for horizontal system 5, with a grid step of $100 \mathrm{~m}$, for a noise standard deviation equal to $10 \%$ of the peak-to-peak amplitude of the components. The color scale is the same for all tensor components. Black lines delineate projections of the top sides of the anomalous bodies onto the $(x, y)$-plane. 
involved are corrected to the same extent $\left(\lambda^{\max }=0.50\right)$ regardless of which equation is kept. This stands for system 7 if we keep the first equation and drop the equation involving $g_{z}$. Instead, if we keep the equation involving $g_{z}$ and drop the first equation, we obtain $\lambda^{\max }$ $=1.00$ for $g_{z}$ and $\lambda^{\max }=0.00$ for $T_{x z}$. Gravity gradients do not seem to benefit from any information brought by $g_{z}$. Therefore, we conclude that as long as data have a sufficient resolution, we should remove two-thirds of the random noise they contain.

We observed in our simulations that given a grid resolution, the $\lambda_{\text {lim }}$ value does not change when the noise level increases, staying equal to two-thirds. We focus later on the behavior of our method as the data-grid sampling decreases because this is related to the frequency content of the sampled signal.

\section{Signal-to-noise ratio}

We investigate the impact of raising or lowering the noise level with a given grid step. As shown, optimal values are obtained for a grid step less than or equal to $200 \mathrm{~m}$. Consequently, the following tests are performed on a regular square grid with a step of $200 \mathrm{~m}$. Results are shown in Figure 4. The method becomes more effective as
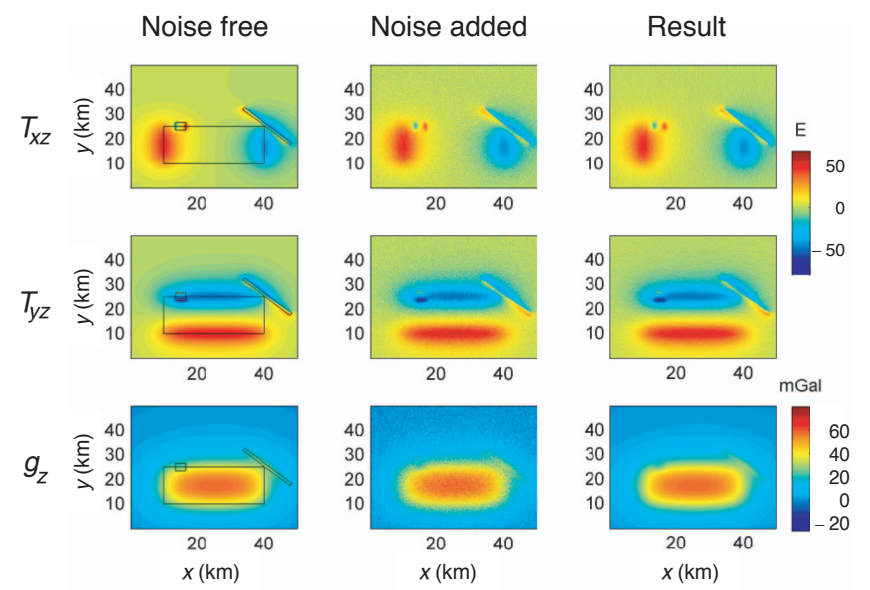

Figure 3. Noise-reduction results for vertical system 7, with a grid step of $100 \mathrm{~m}$, for a noise standard deviation equal to $10 \%$ of the peak-to-peak amplitude of the components. The color scale is the same for all tensor components and, for $g_{z}$, with or without noise and reconstructed. Black lines delineate projections of the top sides of the anomalous bodies onto the $(x, y)$-plane.

Table 2. Noise reduction as a function of the grid step for regular square grids. Results of our method are given for a white Gaussian noise with standard deviation of approximately $10 \%$ of the peak-to-peak amplitude of the component variation.

$\lambda$

\begin{tabular}{lcccccc}
$\begin{array}{l}\text { Grid } \\
\text { step } \\
(\mathrm{m})\end{array}$ & $T_{x x}$ & $T_{x y}$ & $T_{y y}$ & $T_{x z}$ & $T_{y z}$ & $g_{z}$ \\
\hline 1000 & 0.57 & 0.78 & 0.55 & 0.49 & 0.50 & 0.92 \\
500 & 0.59 & 0.78 & 0.59 & 0.50 & 0.49 & 0.98 \\
200 & 0.59 & 0.80 & 0.60 & 0.50 & 0.50 & 0.99 \\
100 & 0.60 & 0.80 & 0.60 & 0.50 & 0.50 & 1.00 \\
\hline
\end{tabular}

the signal-to-noise ratio decreases, and it does not perform well when the noise level is too low. This is thought to be related to (a) the criteria used for stopping the least-squares inversion (see Paige and Saunders (1982) for details on the inversion algorithm used here) and (b) the measurement error becoming small with respect to the discretization error. As the noise level increases, the noise-reduction factor $\lambda$ on each component reaches its optimum value, leading to a mean noise-reduction factor of two-thirds.

\section{Impact of smoothing}

Low-pass filtering is designed to attenuate the high-frequency part of a signal. In this section, we compare the results of our method with a low-pass filter. A Gaussian smoothing (Wells, 1986) with a standard deviation of 0.65 is applied to the gridded data in a $3 \times 3$ point window (Figure 5 for horizontal system 5; Figure 6 for vertical system 7). Of course, better filters could be used. However, because we only want to demonstrate how our method handles high frequencies, this simple filter suffices.

With Gaussian smoothing, $\lambda$ tends asymptotically toward a value close to 0.8 for all components. For $T_{x x}, T_{y y}, T_{x z}$, and $T_{y z}$, Gaussian smoothing performs better than our method when noise increases, but for $T_{x y}$, both methods perform similarly. For $g_{z}$, our method gives the best result. For all components, a combination of smoothing and our method gives better results than using either method alone. We show later through spectral analyses that smoothing and our method have distinct and complementary natures; this is particularly interesting at high frequencies. Note that for lower values of the standard deviation of the noise, $\lambda$ is negative for smoothing. This means that after smoothing, the data fit the noise-free synthetic data less well than without smoothing.

To conclude, we have shown on synthetic data that our method allows us to remove two-thirds of the added noise independently of the noise level as long as the noise is not too small. In our test cases, Gaussian filtering performs slightly better than our method. It removes the noise according to hypotheses on the spectral content of the signal. On the other hand, our method reduces noise on the basis of the potential basis of the tensor. Consequently, filtering and our
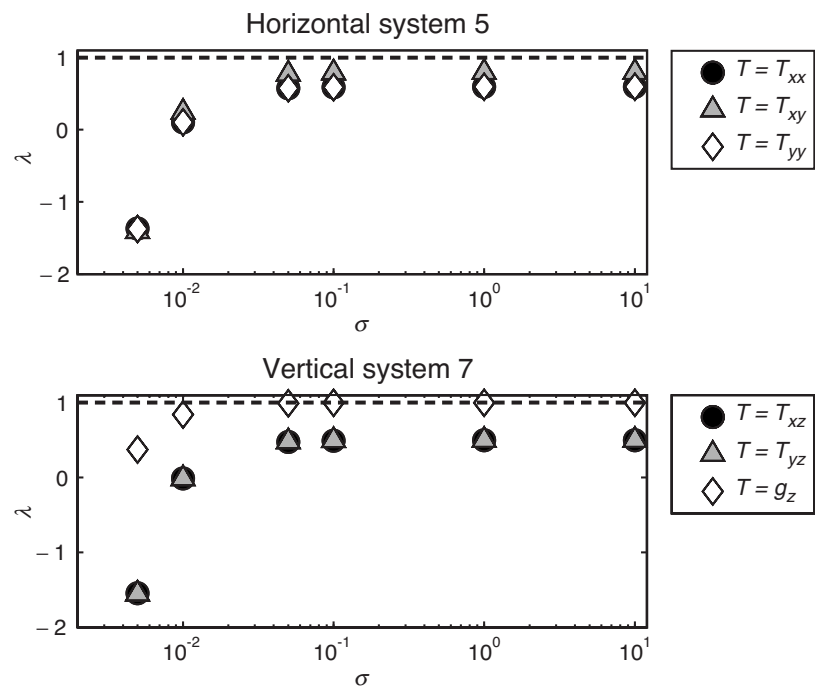

Figure 4. Variations of $\lambda$ according to the logarithm of the noise standard deviation $\sigma$, in Eötvös for the gravity gradients and in milligals for $g_{z}$, on a regular square grid with a step of $200 \mathrm{~m}$. 
method can be used together to optimize noise reduction.

We also tested our method on synthetic data contaminated with covariant noise generated from Gaussian distributions by a secondorder autoregressive process as well as uniformly distributed (nonGaussian) noise. We obtained similar results as with Gaussian white noise (as long as the noise autocorrelation is not unrealistically high in the case of a covariant noise).

\section{Short-wavelength preservation}

As outlined in the introduction, gravity gradients can help retrieve the shortest wavelengths of the gravity signal when these cannot be measured precisely by gravimeters. Therefore, a gradiometry noisereduction method must preserve the high-frequency content. In this respect, our method complements smoothing, as illustrated by the following example.

a) $T_{x x}$

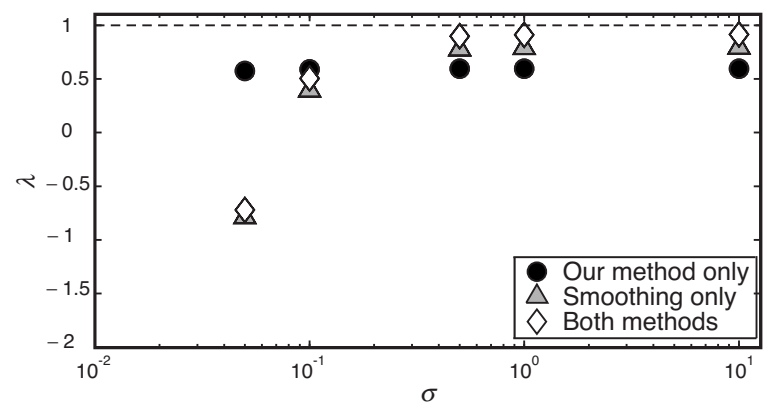

b)

$T_{x y}$

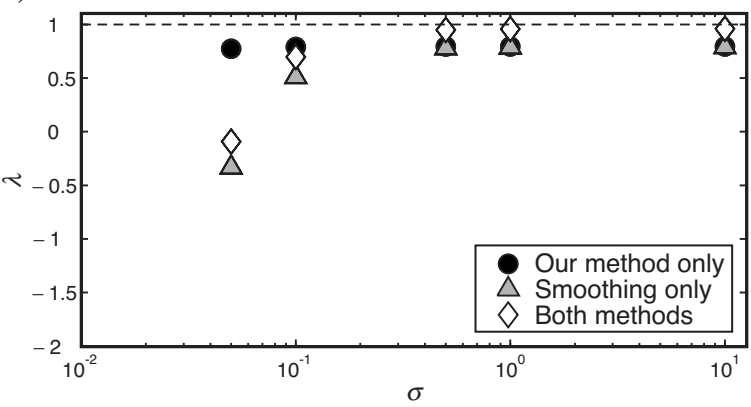

c)

$T_{y y}$

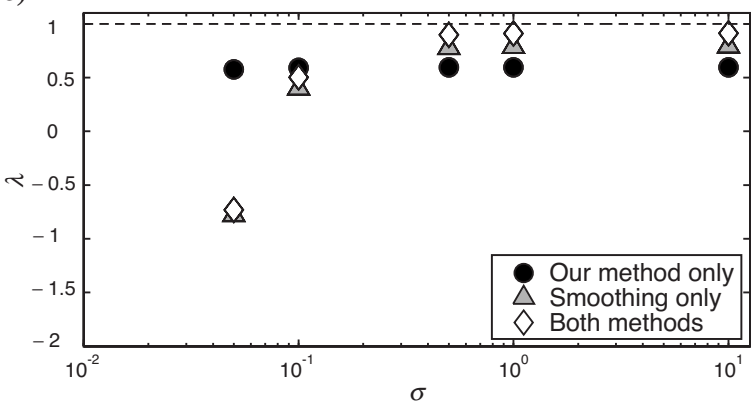

Figure 5. Comparison, on a regular square grid with a step of $200 \mathrm{~m}$, between the impact of Gaussian smoothing in a $3 \times 3$ point window and our noise-reduction method for (a) the tensor component $T_{x x}$, (b) the tensor component $T_{x y}$, and (c) the tensor component $T_{y y}$ involved in horizontal system 5 . The circle stands for the result of our method only, the triangle for the result of Gaussian smoothing only, and the diamond for the result of the two methods applied successively. The standard deviation $\sigma$ is in Eötvös.
We generated a structure composed of 40 identical rectangular prisms measuring $50 \mathrm{~km} \times 50 \mathrm{~m}$ in the $(x, y)$-plane, with depths to their top and bottom sides of $30 \mathrm{~m}$ and $10 \mathrm{~km}$, respectively, and density of $1000 \mathrm{~kg} / \mathrm{m}^{3}$. Twenty prisms were regularly set parallel to the $y$-axis every $2.4 \mathrm{~km}$. These were intersected by 20 other prisms, regularly set parallel to the $x$-axis every $2.4 \mathrm{~km}$. The gradient contributions of the 40 prisms were added so that their intersections (rectangular prisms with square $-50-\times 50$-m top sides) had twice the density of the individual prisms. The projection of this structure onto the $(x, y)$-plane is shown in Figure 7.

We computed the $g_{z}$ signal on three square $50 \times 50$-km grids, with data points every 250,500 , and $1000 \mathrm{~m}$. We contaminated $g_{z}$ with $10 \%$ Gaussian noise. For the grids that had steps of 250 and $500 \mathrm{~m}$, the $\lambda$-parameter for our method was greater than 0.99 , which is always greater than the maximum $\lambda$ value (0.94) obtained with smoothing (the best window size for smoothing is, according to our tests, $7 \times 7$ for 250 -m step grids and $5 \times 5$ for 500 -m step grids).
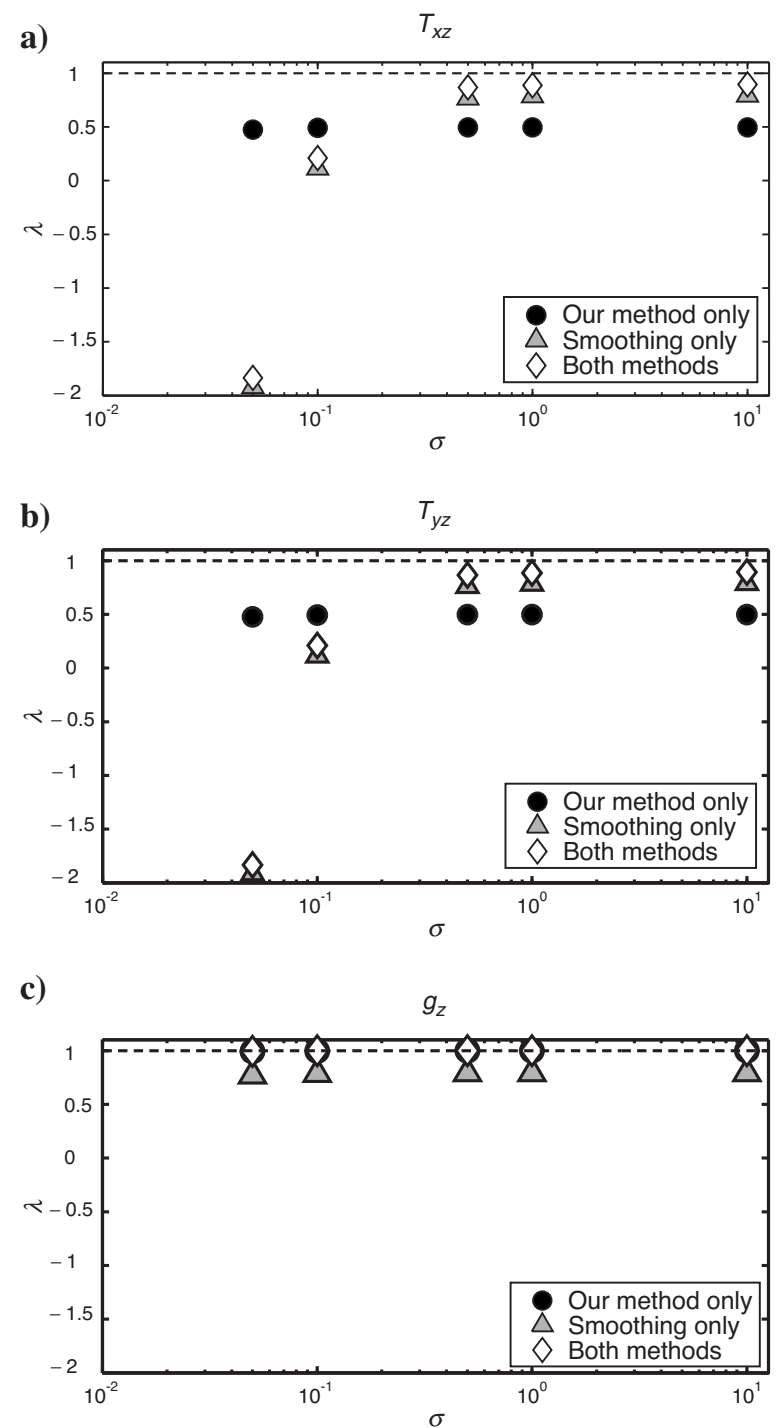

Figure 6. Comparison, on a regular square grid with a step of $200 \mathrm{~m}$, between the impact of Gaussian smoothing in a $3 \times 3$ point window and our noise-reduction method for (a) the tensor component $T_{x z}$, (b) the tensor component $T_{y z}$, and (c) the component $g_{z}$ involved in vertical system 7 . 


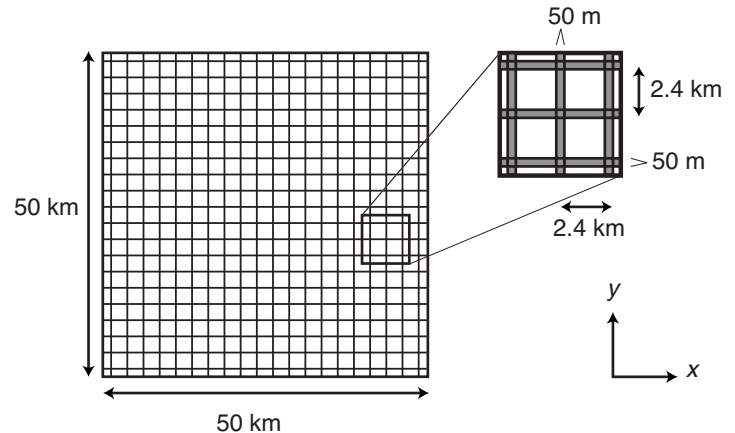

Figure 7. Projection onto the $(x, y)$-plane of the structure used to show how our method handles small wavelengths. The structure is made of 40 identical rectangular prisms arranged in an orthogonal lattice, each prism having $(x, y)$-dimensions of $50 \mathrm{~m} \times 50 \mathrm{~km}$. The depth to the top sides of the prism is $30 \mathrm{~m}$. The depth to the bottom is $10,000 \mathrm{~m}$. The density contrast is $1000 \mathrm{~kg} / \mathrm{m}^{3}$.

a)

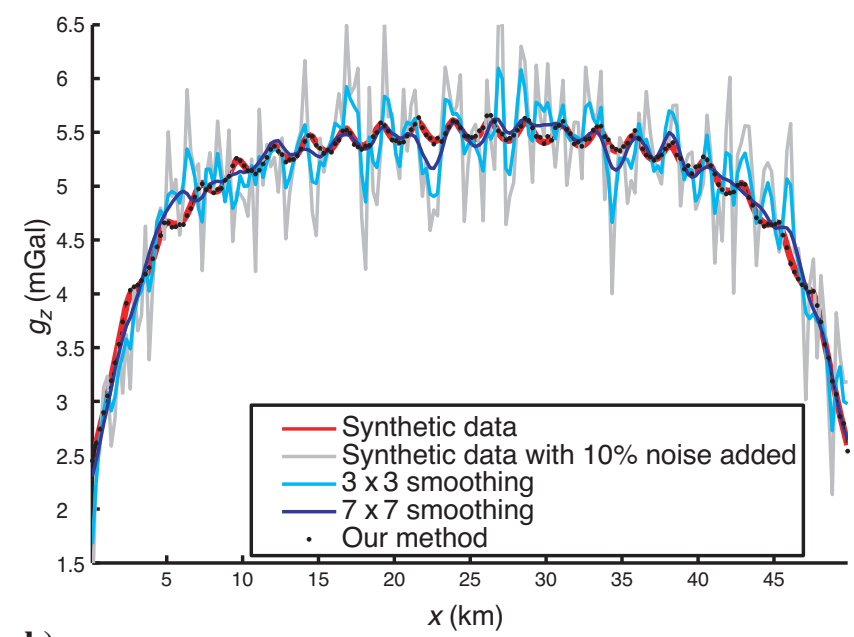

b)

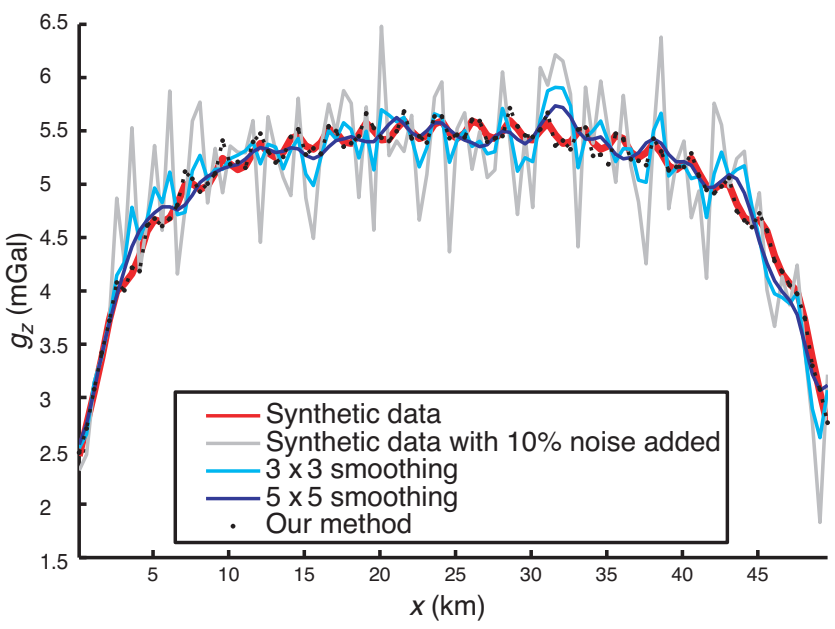

Figure 8. Comparison along a profile for a grid step of (a) $250 \mathrm{~m}$ and (b) $500 \mathrm{~m}$ between the noise-reduction results of our method and Gaussian smoothing with varying window size, applied on $g_{z}$. In the background, gray represents the anomaly with $10 \%$ noise added, red the original anomaly, dotted black our result, and dark and light blue the results of Gaussian smoothing.
Results on a profile for the 250-m step grid and the 500-m step grid are shown in Figure 8. The best result obtained by Gaussian smoothing does not fit the original noise-free synthetic data as well as our solution does. The average power spectra in the $x$-direction (mean of the spectra along all profiles parallel to the $x$-axis) for these two examples are shown in Figure 9. For the 250-m step grid, the power curve of our solution (dotted black) matches almost exactly that of the original data (red). At the larger grid step, our solution has too much power in the shorter wavelengths (high frequencies), but its spectrum still has a shape similar to that of the original data.

Tests performed at a grid step of $1000 \mathrm{~m}$ confirm that when approaching the Nyquist frequency $\left(0.84=1 / 1.19 \mathrm{~km}^{-1}\right)$, power in the high frequencies is amplified (see Figure 10). The anomalies are detected better than with smoothing, but their magnitude is overestimated. It then becomes necessary to apply a light smoothing $(3 \times 3)$ to our solution to retrieve the original signal, but with greater accuracy than with smoothing only. This example shows that smoothing and our method complement each other, especially for short-wavelength signals.
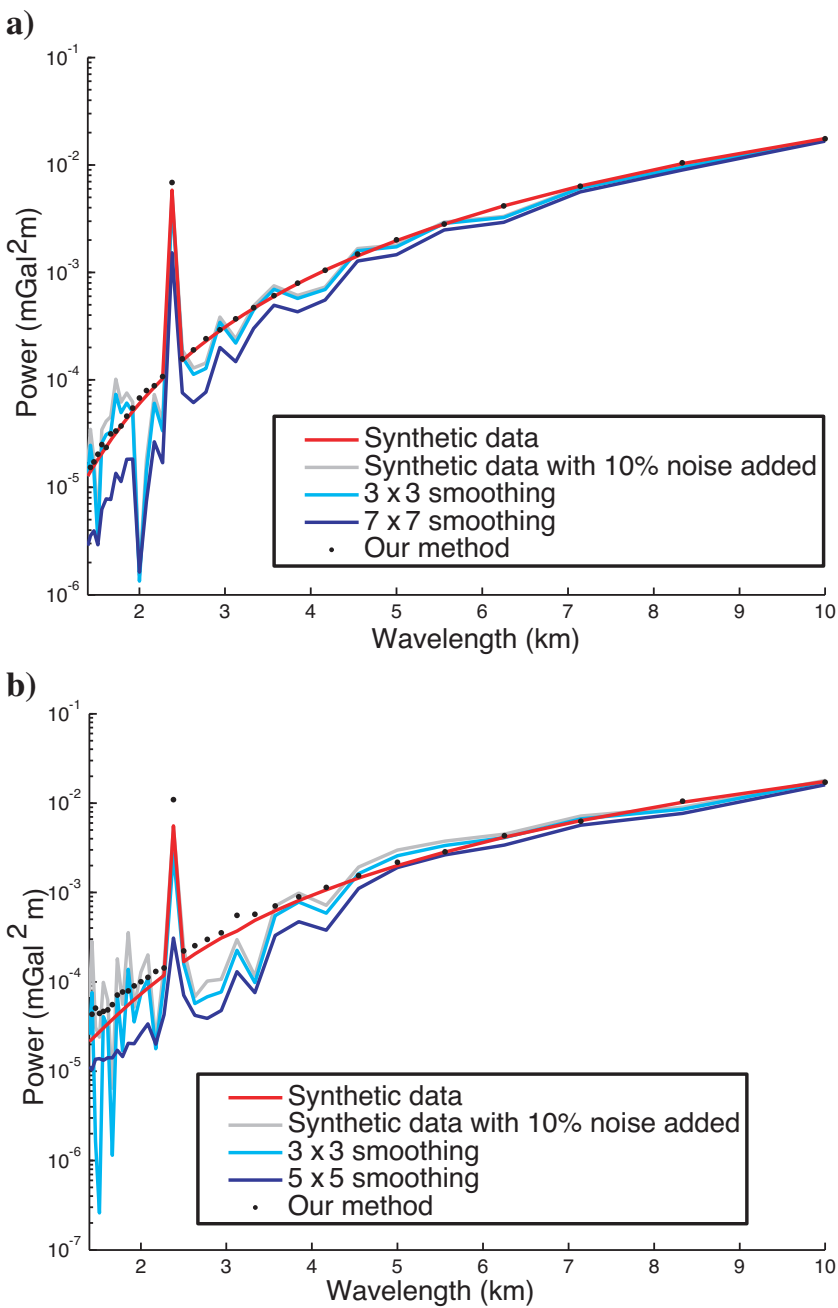

Figure 9. Mean of the power spectra of all profiles parallel to the $x$-axis for the results of our method and for Gaussian smoothing on $g_{z}$ for a grid step of (a) $250 \mathrm{~m}$ and (b) $500 \mathrm{~m}$. Colors are the same as for Figure 8. 


\section{APPLICATION TO MARINE GRAVITY GRADIOMETRY DATA}

We now apply our method to a set of marine gravity gradiometry data and the vertical gravity anomaly collected over the Faroe-Shetland Basin. These data were provided by Bell Geospace for this study. The various components were corrected for bathymetric effects before being provided to us. The data set we consider is a subset of a larger survey, thoroughly described by Mumaw (2004). The survey is composed of roughly parallel lines oriented at $45 \mathrm{~N}$, called L-lines, and lines perpendicular to the L-lines, called T-lines, mapped onto the measured gravity anomaly in Figure 11.

\section{Application to Bell Geospace data}

The tests in Appendix B show that as long as data are distributed on a near-regular lattice, we can work on a regular grid without greatly impacting the result, thus saving computing time. We prepared the data set to be handled in two steps. First, we rotated the grid coordinates so that L-lines are parallel to the $x$-axis, pointing eastward, and T-lines are parallel to the $y$-axis, pointing northward. The gravity gradients are tensor components, so they are transformed to account for the rotation of the coordinate system.

Second, we regularized the grid. We determined a grid made of strictly parallel and perpendicular lines that approaches the original survey grid. We constructed a rectangular lattice with points regularly spaced on an L- or T-line and identified a given lattice point with the nearest survey point. We obtained $29 \mathrm{~L}$-lines separated by $750 \mathrm{~m}$

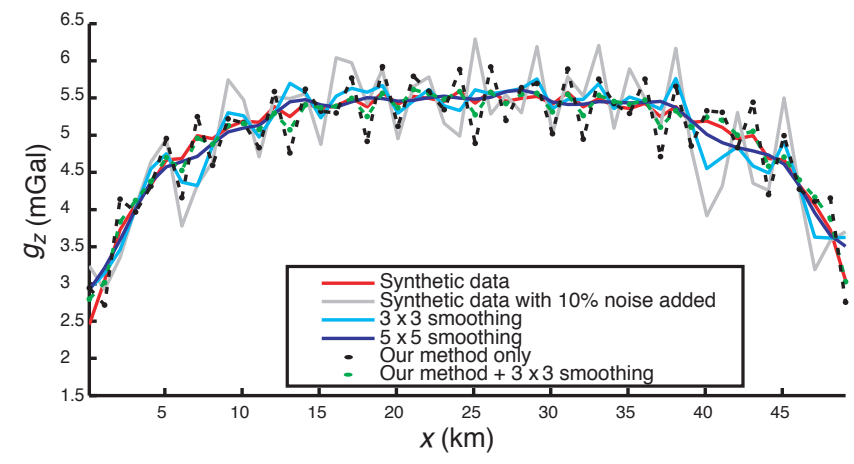

Figure 10. Comparison along a profile for a grid step of $1000 \mathrm{~m}$ between the results of our method enhanced by smoothing and the results of smoothing only.

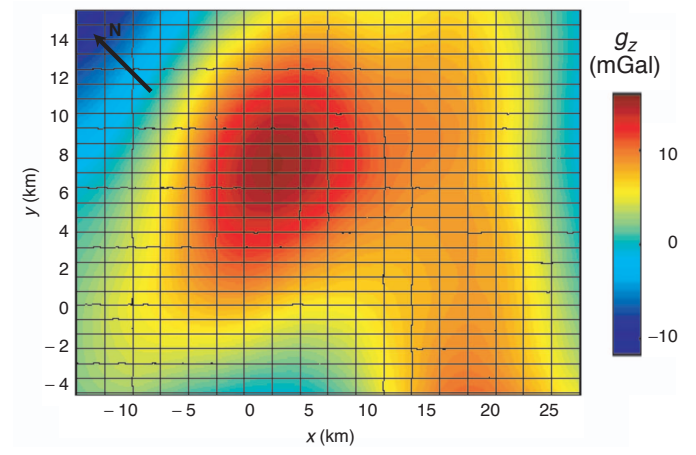

Figure 11. Bell Geospace survey line path mapped onto the gravity data. and $21 \mathrm{~T}$-lines separated from each other by $2250 \mathrm{~m}$. This operation resulted in a grid of 1018 points regularly spaced on the T-lines, with an interval equal to $21 \mathrm{~m}$, and 2105 points on an L-line, with the same interval. We obtained a standard deviation of the position errors of less than $20 \mathrm{~m}$, which is of no significant impact on the result.

\section{Results}

We present the result of our method only, with no additional filtering. Indeed, choosing the appropriate cutoff wavelength for smoothing would require a priori hypotheses on the sources, which is irrelevant here.

Before we applied our noise-reduction method, we tested the raw data for relationships 5 and 7 . Residuals between computed derivatives of $g_{z}$ in the $x$ - and $y$-directions and $T_{x z}$ and $T_{y z}$, respectively, showed no large-scale patterns — only small-scale spatial variations with amplitude of the same magnitude as the signal. After applying the method, this amplitude was reduced significantly (by 50-100\%).

The results are illustrated in Figures 12 and 13. We can see that many short-wavelength features have been removed. However, small-scale features that would have been smoothed by low-pass filtering are preserved. The gravity anomaly $g_{z}$ has been modified slightly. The comparison, on a profile parallel with the $x$-axis, between the noise-reduction result and the original raw data is shown for $T_{y z}$ in Figure 14.

\section{DISCUSSION}

When describing a gravity gradiometer system similar to the Lockheed Martin instrument, van Leeuwen et al. (2005) identify two general types of noise in airborne (or shipborne) gravity gradiometry measurements: deterministic and intrinsic random noise. Determin-
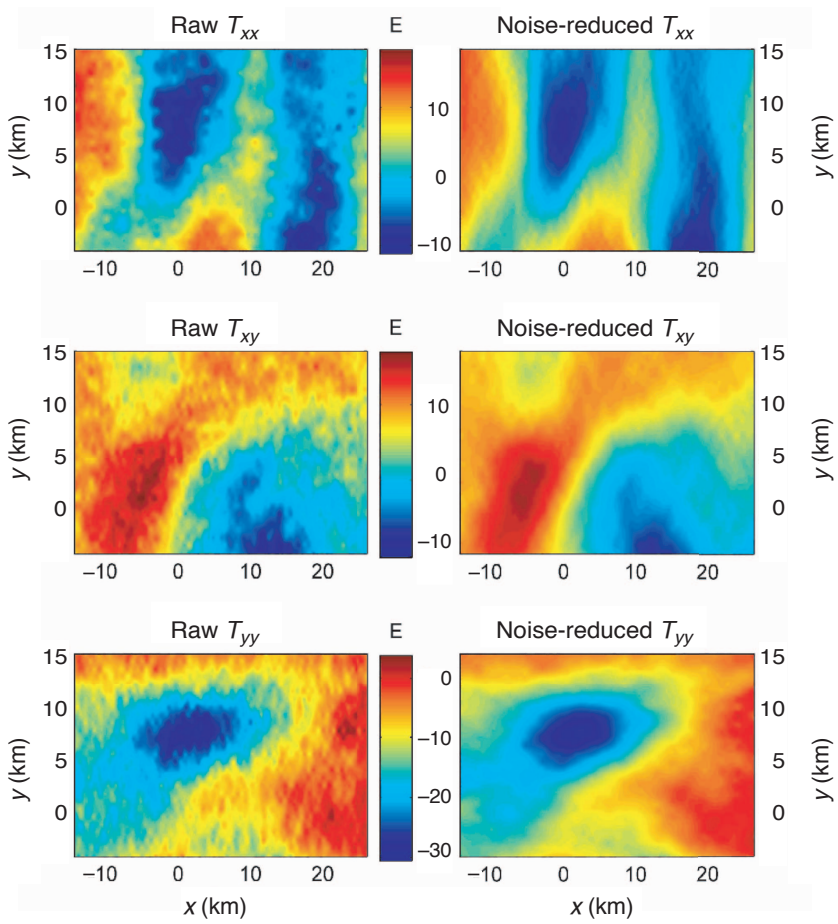

Figure 12. Results of the noise-reduction method applied to Bell Geospace data. Left column: components involved in system 5. Right column: noise-reduction results on these components. 
istic noise has three main causes: (1) the change in gravity sensed by the gradiometer when masses move with respect to the instrument itself (self-gradient); (2) an additional contribution to the signal, called residual acceleration sensitivity, that is proportional to the accelerations measured by the gradiometer; and (3) a contribution from, for instance, the rotation of the platform on which the gradiometer is mounted.

The self-gradient is a true gradient; therefore, it obeys the equations in systems 5 and 7. The same holds true for rotational noise, which mimics a gravity gradient. According to van Leeuwen et al. (2005), these contributions are corrected easily because they can be modeled or measured prior to the survey and removed during or after acquisition. However, intrinsic random noise limits the measurement accuracy and even limits reduction of residual acceleration sensitivity. Being purely random, this intrinsic noise is addressed fully and removed successively by our method.
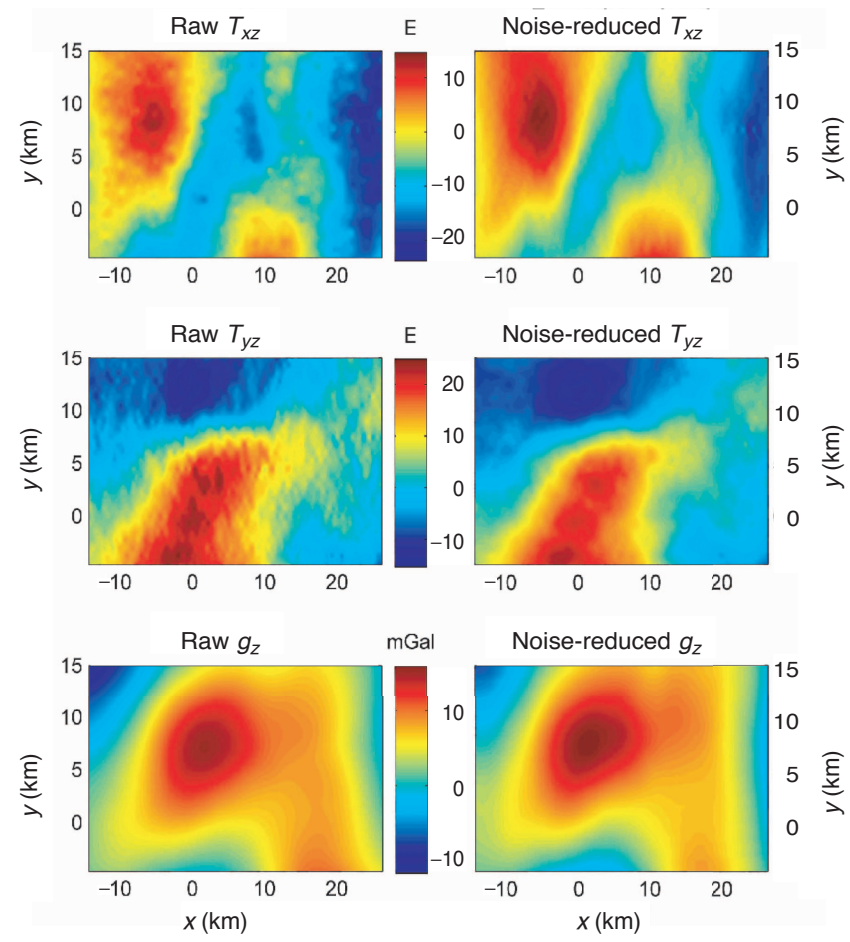

Figure 13. Results of the noise-reduction method applied to Bell Geospace data. Left column: components involved in system 7. Right column: noise-reduction results on these components.

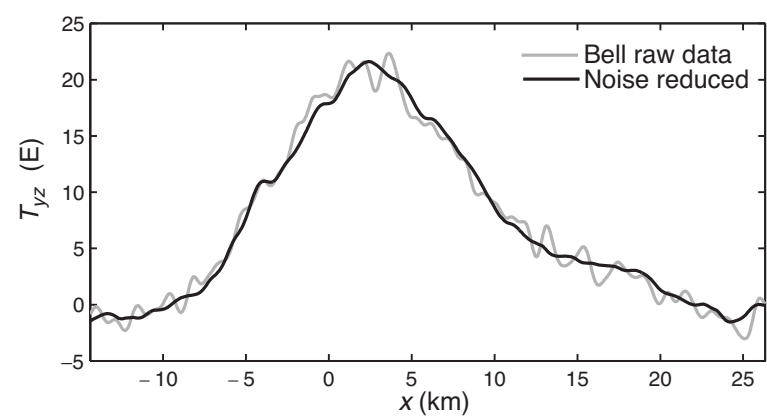

Figure 14. Noise-reduction results on Bell Geospace data along a profile parallel to the $x$-axis for tensor component $T_{y z}$.
In the GOCE mission as well as in airborne surveys, all tensor components are measured simultaneously, but $g_{z}$ is not measured simultaneously. The gravity field is computed from both the measured gradient components, for the high-frequency part, and the precise determination of the orbit of the satellite, for the low-frequency part (Visser and Van den Ijssel, 2000). Our method still can be applied to the components involved in system 5 if a method can be found to compute the $z$-derivative (for example, by using a multisatellite system or upward continuation of the components).

The method even can be refined using, instead of systems 5 and 7 , the physical constraints defined by the following approximate linear differential system:

$$
\left\{\begin{array}{l}
\partial_{y} T_{x x}=\partial_{x} T_{x y} \\
\partial_{y} T_{x y}=\partial_{x} T_{y y} \\
\partial_{y} T_{x z}=\partial_{x} T_{y z} \\
\partial_{z} T_{x x}=\partial_{x} T_{x z} \\
\partial_{z} T_{x y}=\partial_{x} T_{y z} \\
\partial_{z} T_{y y}=\partial_{y} T_{y z} \\
\partial_{y} T_{z z}=\partial_{z} T_{y y} \\
\partial_{x} T_{z z}=\partial_{z} T_{x z}
\end{array} .\right.
$$

The vertical component $T_{z z}$ actually is measured rather than being computed from $T_{x x}$ and $T_{y y}$, so Laplace's equation also can be added.

\section{CONCLUSION}

We have developed a method for simultaneously reducing noise from independent gravity and FTG data. This method does not require a priori information. Moreover, it uses all measured values simultaneously instead of handling lines of the survey separately. Therefore, all available information coming from the data is used to improve the values at each measurement point. We do not filter the data, so the high frequencies of the signal are preserved. This is of tremendous importance when dealing with such data because the expected benefit from gravity gradiometry data is the additional highfrequency information brought by the derivatives, as compared with gravity measurements only. Our method corrects half of the random noise affecting gravity gradients (which later can be improved by light filtering if needed for gradiometry interpretation) and most of the noise in the gravity anomaly.

Furthermore, results are affected by noise autocorrelation only weakly. Finite differences can be replaced by another way to compute the derivatives as long as the derivative can be written as a linear combination of some values of the components. However, this increases the computing time (from 3 to $17 \mathrm{~s}$ for the Bell Geospace data example on a standard personal computer) and is not necessary for low-resolution data. We demonstrated the power of this method on synthetic data and illustrated it on real data acquired by Bell Geospace over the Faroe-Shetland Basin. We obtained maps showing that noise was reduced visibly and that small-scale features were preserved that would have been smoothed out by a filtering method.

In the Bell Geospace data, the diagonal components of the tensor were preprocessed so the trace was zero. Consequently, only two of these diagonal components have independent errors, so our method cannot be applied using the three diagonal components simultaneously. We chose not to solve for $T_{z z}$; because $g_{z}$ was in our case 
study, we had another option to remove noise from the nondiagonal components. Noise reduction likely will improve if we add to the horizontal and vertical system equations involving $T_{z z}$ when it is measured simultaneously with the other gradients.

\section{ACKNOWLEDGMENTS}

We gratefully acknowledge Bell Geospace (and, in particular, Colm Murphy) for making marine gravity gradiometry data available for our study. We also thank Maria Annechione, Des Fitzgerald, Horst Holstein, Xiong Li, and anonymous reviewers for their careful reading of this manuscript and for their helpful remarks. G. Pajot benefited from a DGA grant, and the whole work was supported by the French space agency Centre National d'Études Spatiales (CNES) through the Tosca committee. We thank M. Van Camp and M. Beuthe for discussion and helpful comments. This is Institut de Physique du Globe de Paris (IPGP) contribution 2330.

\section{APPENDIX A}

\section{COMPUTING THE DERIVATIVES}

The equations we used throughout the study are of the form

$$
\frac{\partial F(x, y)}{\partial x}-\frac{\partial G(x, y)}{\partial y}=0
$$

or, in the case of system 7 , of the form

$$
\frac{\partial F(x, y)}{\partial x}-G(x, y)=0
$$

or

$$
\frac{\partial F(x, y)}{\partial y}-G(x, y)=0 .
$$

Let us focus on the form described by equation A-1. This can be approximated by the centered-difference scheme:

$$
\frac{F\left(x_{i+1}, y_{j}\right)-F\left(x_{i-1}, y_{j}\right)}{\Delta x}=\frac{G\left(x_{i}, y_{j+1}\right)-G\left(x_{i}, y_{j-1}\right)}{\Delta y} \text {. }
$$

This scheme becomes increasingly unfavorable when the sampling interval decreases if the signal does not include enough high-wavenumber content. This is obviously the case in our simulation because of the simplicity and lateral extent of the sources in consideration.

Let us suppose that the sampling interval decreases in the $x$-direction $(\Delta x \ll \Delta y)$. To overcome this problem, we apply the equation linking the gradient to what would be the best polynomial fit of the data, with given degree, in direction $x$ of the highest sampling. The approximation of the $2 N+1$ measurements $F\left(x_{k}, y_{j}\right), k$ $=i-N \ldots i+N$ using a polynomial of degree $n$ at locations $x_{k}$ can be written as

$$
F\left(x_{k}, y_{j}\right) \simeq \sum_{m=0}^{n} \alpha_{m} x_{k}^{m}
$$

where the coefficients $\alpha_{i}$ are determined so that the sum of the squared residuals is minimal. We determine the expression of the $\alpha_{i}$ coefficients as a function of the value of the datum $F\left(x_{k}, y_{j}\right)$, and we substitute them in the expression of the derivative of the polynomial fit:

$$
\frac{\partial F\left(x_{i}, y_{j}\right)}{\partial x} \simeq \sum_{m=1}^{n} m \alpha_{m} x_{i}^{m-1} .
$$

Every $\alpha_{m}$ can be expressed as a linear combination of the measurements $F\left(x_{k}, y_{j}\right)$, so we then obtain an expression such as

$$
\frac{\partial F\left(x_{i}, y_{j}\right)}{\partial x} \simeq \sum_{m=i-N}^{i+N} \beta_{m} F\left(x_{m}, y_{j}\right)
$$

where $\beta_{m}$ coefficients depend on $1 / \Delta x$. Consequently, in the leastsquares fit of our data, we replace equation A-4 with

$$
\sum_{m=i-N}^{i+N} \beta_{m} F\left(x_{m}, y_{j}\right)-\frac{G\left(x_{i}, y_{j+1}\right)-G\left(x_{i}, y_{j-1}\right)}{\Delta y}=0 .
$$

Similarly, equation A-2 transforms to

$$
\sum_{m=i-N}^{i+N} \beta_{m} F\left(x_{m}, y_{j}\right)-G\left(x_{i}, y_{j}\right)=0
$$

but equation A-3 stays as

$$
\frac{F\left(x_{i}, y_{j+1}\right)-F\left(x_{i}, y_{j-1}\right)}{\Delta y}-G\left(x_{i}, y_{j}\right)=0 .
$$

\section{APPENDIX B}

\section{EFFECT OF GRID IRREGULARITIES}

In our tests, we used regular square grids with identical steps in the $x$ - and $y$-directions. To handle real data, we must account for two main differences: (1) the sampling interval along a line is much smaller than the distance between lines, and (2) the positions of the measurement points do not follow straight lines.

\section{Grid anisotropy}

At first, we work on a grid where data are distributed on a lattice made of parallel and perpendicular straight lines, but we investigate what happens for the points not located where lines intersect. We begin with the synthetic structure, a $50-\times 50-\mathrm{km}$ square grid with a noise level equal to about $10 \%$ of the maximum peak-to-peak amplitude of the component. In this case, where the interval between two consecutive points on a profile is small, finite differences are not appropriate for computing the derivatives when handling real data. We performed tests with several orders of finite-difference schemes with no significant improvement in the results. We present here what happens when the sampling interval decreases in one direction.

Tables B-1 and B-2 summarize the noise-reduction results when the sampling interval decreases in the $y$ - and $x$-directions, respectively. In both cases, the derivatives are computed using centered second-order finite differences (see Appendix A). The derivative along the direction in which the sampling interval decreases $\left(T_{x x}\right.$ and $T_{x z}$ for $y$-interval decreasing, $T_{y y}$ and $T_{y z}$ for $x$-interval decreasing) or 
Table B-1. Variations of the $\lambda$-parameter, for a noise level equal to $10 \%$ of the maximum peak-to-peak amplitude of the component for decreasing sampling interval along the $y$-axis. The interval along the $x$-axis is constant $(1 \mathrm{~km})$.

\begin{tabular}{lcccccc} 
& \multicolumn{7}{c}{$\lambda$ values $(d x=1 \mathrm{~km})$} \\
\cline { 2 - 7 } Component & $\begin{array}{c}d y=1000 \\
\mathrm{~m}\end{array}$ & $\begin{array}{c}d y=500 \\
\mathrm{~m}\end{array}$ & $\begin{array}{c}d y=250 \\
\mathrm{~m}\end{array}$ & $\begin{array}{c}d y=125 \\
\mathrm{~m}\end{array}$ & $\begin{array}{c}d y=62.5 \\
\mathrm{~m}\end{array}$ & $\begin{array}{c}d y=31.25 \\
\mathrm{~m}\end{array}$ \\
\hline$T_{x x}$ & 0.55 & 0.76 & 0.85 & 0.90 & 0.92 & 0.93 \\
$T_{x y}$ & 0.78 & 0.83 & 0.89 & 0.94 & 0.96 & 0.97 \\
$T_{y y}$ & 0.53 & 0.36 & 0.17 & 0.07 & 0.03 & 0.01 \\
$T_{x z}$ & 0.48 & 0.68 & 0.82 & 0.89 & 0.93 & 0.95 \\
$T_{y z}$ & 0.50 & 0.26 & 0.13 & 0.06 & 0.03 & 0.01 \\
$g_{z}$ & 0.97 & 0.99 & 0.99 & 1.00 & 1.00 & 1.00 \\
\hline
\end{tabular}

Table B-2. Variations of the $\lambda$-parameter, for a noise level equal to $10 \%$ of the maximum peak-to-peak amplitude of the component for decreasing sampling interval along the $x$-axis. The interval along the $y$-axis is constant $(1 \mathrm{~km})$.

\begin{tabular}{lcccccc} 
& \multicolumn{7}{c}{$\lambda$ values $(d y=1 \mathrm{~km})$} \\
\cline { 2 - 7 } Component & $\begin{array}{c}d x=1000 \\
\mathrm{~m}\end{array}$ & $\begin{array}{c}d x=500 \\
\mathrm{~m}\end{array}$ & $\begin{array}{c}d x=250 \\
\mathrm{~m}\end{array}$ & $\begin{array}{c}d x=125 \\
\mathrm{~m}\end{array}$ & $\begin{array}{c}d x=62.5 \\
\mathrm{~m}\end{array}$ & $\begin{array}{c}d x=31.25 \\
\mathrm{~m}\end{array}$ \\
\hline$T_{x x}$ & 0.55 & 0.34 & 0.16 & 0.07 & 0.02 & 0.01 \\
$T_{x y}$ & 0.78 & 0.82 & 0.89 & 0.94 & 0.96 & 0.97 \\
$T_{y y}$ & 0.53 & 0.75 & 0.85 & 0.89 & 0.92 & 0.93 \\
$T_{x z}$ & 0.48 & 0.29 & 0.14 & 0.07 & 0.03 & 0.01 \\
$T_{y z}$ & 0.50 & 0.67 & 0.80 & 0.88 & 0.92 & 0.94 \\
$g_{z}$ & 0.97 & 0.98 & 0.99 & 1.00 & 1.00 & 1.00 \\
\hline
\end{tabular}

free synthetic data $T^{1}$ computed at $\left(x_{i}^{1}, y_{j}^{1}\right)($ test $\mathrm{A})$ and to the noise-free synthetic data $T^{0}$ computed at $\left(x_{i}^{0}, y_{j}^{0}\right)$ (test B).

We performed this test with the same synthetic data used above with $10 \%$ noise added on the components, using a regular square grid with a 250-m sampling interval. For both anomalous bodies, we added a random normal error with a 25-m standard deviation (corresponding to our data set) on the $x$ - and $y$-coordinates. The results are almost identical to those obtained for regular square grids. The $\lambda$-parameters are slightly better (approximately 0.01 greater) for test B than for test $\mathrm{A}$, which might indicate that in the examples we studied, our method handles noise in coordinates as noise in components. However, the difference is too small to be considered statistically significant.

\section{REFERENCES}

Bell, R. E., R. Anderson, and L. F. Pratson, 1997, Gravity gradiometry resurfaces: The Leading Edge, 16 , $55-59$

Canuto, E., P. Martella, and G. Sechi, 2003, Attitude and drag control: An application to the GOCE satellite: Space Science Reviews, 108, 357-366.

Dransfield, M. H., and J. B. Lee, 2004, The FALCON airborne gravity gradiometer survey systems: Australian Society of Exploration Geophysicists/Petroleum Exploration Society of Australia (ASEGPSEA) Airborne Gravity Workshop, Expanded Abstracts, 15-20.

European Space Agency, 1999, Gravity field and steady-state ocean circulation mission: Technical report SP-1233(1).

Hammond, S., 1999, Acquiring and processing gradient gravity data: Offshore, 59,92-93.

Hofmeyer, G., and C. Affleck, 1994, Rotating accelerometer gradiometer: U. S. Patent 5357802.

Klees, R., R. Koop, P. Visser, and J. Van den Ijssel,

components that have derivatives involved in more than one equation $\left(T_{x y}\right.$ and $\left.g_{z}\right)$ all are corrected better because the sampling interval decreases in either of the two directions. However, at the same time, the noise affecting the other components is less well reduced.

The problem is overcome when using, to calculate derivatives, a least-squares fit of a low-degree polynomial, as described in Appen$\operatorname{dix}$ A. Results are improved in such a way that we retrieve results similar to those obtained on regular square grids. However, this multiplies the computing time by five and can be avoided when unnecessary.

\section{Positioning misfit}

To test what happens for data that are not distributed perfectly along straight lines, we performed the following test. First we let $\left(x_{i}^{0}, y_{j}^{0}\right)$ be the coordinates of the measurement points on a regular square grid. A random component with normal distribution was added to the coordinates of each of these points. We denoted $\left(x_{i}^{1}, y_{j}^{1}\right)$ as the new coordinates. Next we generated synthetic data. The values $T^{1}$ of the components were computed at $\left(x_{i}^{1}, y_{j}^{1}\right)$. Third, we added noise to the values of the components and then applied our method to the data, assuming the measurement points to be $\left(x_{i}^{0}, y_{j}^{0}\right)$ instead of $\left(x_{i}^{1}, y_{j}^{1}\right)$. Finally, we compared the results of our method to the noise2000, Efficient gravity field recovery from GOCE gravity gradient observations: Journal of Geodesy, 74, 561-571.

Lyrio, J. C. S., L. Tenorio, and Y. Li, 2004, Efficient automatic denoising of gravity gradiometry data: Geophysics, 69, 772-782.

Mikhailov, V., G. Pajot, M. Diament, and A. Price, 2007, Tensor deconvolution: A method to locate equivalent sources from full tensor gravity data: Geophysics, 72, no. 5, I61-I69.

Mumaw, G., 2004, Marine 3D full tensor gravity gradiometry — The first five years: Hydro International, 8, 38-41.

Okabe, M., 1979, Analytical expressions for anomalies due to polyhedral bodies and translation into magnetic anomalies: Geophysics, 44, 730-741.

Paige, C. C., and M. A. Saunders, 1982, LSQR, an algorithm for sparse linear equations and sparse least squares: Association for Computing Machinery Transactions on Mathematical, Software, 8, 43-71.

Shaw, H., and B. Lancaster-Jones, 1922, The Eötvös torsion balance: Proceedings of the Physical Society of London, 35, 151-166.

Szabó, Z., 1998, Eötvös the man, the scientist, the organizer: Eötvös Loránd Geophysical Institute.

van Leeuwen, E. H., K. G. McCracken, J. B. Lee, and R. J. Turner, 2005, Airborne gravity gradiometer: U. S. Patent $6883372 \mathrm{~B} 2$.

Visser, P., and J. Van den Ijssel, 2000, GPS-based precise orbit determination of the very low earth-orbiting gravity mission GOCE: Journal of Geodesy, 74, 590-602.

Wells, W., 1986, Efficient synthesis of Gaussian filters by cascaded uniform filters: IEEE Transactions on Pattern Analysis and Machine Intelligence, PAMI-8, 234-239.

While, J., A. Jackson, D. Smit, and E. Biegert, 2006, Spectral analysis of gravity gradiometry profiles: Geophysics, 71, 11-22. 